\title{
PROMIS Pediatric and Parent Proxy Physical Health Domain
}

National Cancer Institute

\section{Source}

National Cancer Institute. PROMIS Pediatric and Parent Proxy Physical Health Domain.

NCl Thesaurus. Code C156219.

The set of PROMIS measures that assess the physical health of pediatric subjects

through self-report or parent proxy report. 